\title{
Treatment of Allergic Asthma with Fenretinide Formulation (LAU-7b) Downregulates ORMDL Sphingolipid Biosynthesis Regulator 3 (Ormd/3) Expression and Normalizes Ceramide Imbalance ${ }^{\mathrm{g}}$
}

\author{
Mina Youssef, Juan B. De Sanctis, Juhi Shah, Daciana Catalina Dumut, Marian Hajduch, \\ Anna K. Naumova, and (DDanuta Radzioch \\ Department of Human Genetics (M.Y., A.K.N., D.R.), Department of Pharmacology and Therapeutics (J.S.), Division of \\ Experimental Medicine, Department of Medicine (D.C.D., D.R.), and Department of Obstetrics and Gynecology (A.K.N.), McGill \\ University, Montreal, Quebec, Canada; Program in Infectious Diseases and Immunity in Global Health, McGill University Health \\ Center, Montreal, Quebec, Canada (M.Y., J.S., D.C.D., D.R.); and Institute of Molecular and Translational Medicine, Faculty of \\ Medicine and Dentistry, Palacky University, Olomouc, Czech Republic (J.B.D.S., M.H., D.R.)
}

Received November 6, 2019; accepted February 5, 2020

\begin{abstract}
Zona pellucida binding protein 2 (Zpbp2) and ORMDL sphingolipid biosynthesis regulator 3 (Ormd/3), mapped downstream of Zpbp2, were identified as two genes associated with airway hyper-responsiveness (AHR). Ormdl3 gene product has been shown to regulate the biosynthesis of ceramides. Allergic asthma was shown to be associated with an imbalance between very-longchain ceramides (VLCCs) and long-chain ceramides (LCCs). We hypothesized that Fenretinide can prevent the allergic asthmainduced augmentation of Ormdl3 gene expression, normalize aberrant levels of VLCCs and LCCs, and treat allergic asthma symptoms. We induced allergic asthma by house dust mite (HDM) in AVJ WT mice and Zpbp2 KO mice expressing lower levels of Ormdl3 mRNA than WT. We investigated the effect of a novel formulation of Fenretinide, LAU-7b, on the AHR, inflammatory cell infiltration, mucus production, IgE levels, and ceramide levels. Although lower Ormdl3 expression, which was observed in Zpbp2 $\mathrm{KO}$ mice, was associated with lower AHR, allergic Zpbp2 KO mice were not protected from inflammatory cell infiltration, mucus accumulation, or aberrant levels of VLCCs and LCCs induced by
\end{abstract}

HDM. LAU-7b treatment protects both the Zpbp2 KO and WT mice. The treatment significantly lowers the gene expression of Ormdl3, normalizes the VLCCs and LCCs, and corrects all the other phenotypes associated with allergic asthma after HDM challenge, except the elevated levels of IgE. LAU-7b treatment prevents the augmentation of Ormdl3 expression and ceramide imbalance induced by HDM challenge and protects both WT and Zpbp2 KO mice against allergic asthma symptoms.

\section{SIGNIFICANCE STATEMENT}

Compared with A/J WT mice, $\mathrm{KO}$ mice with Zpbp2 gene deletion have lower AHR and lower levels of Ormdl/3 expression. The novel oral clinical formulation of Fenretinide (LAU-7b) effectively lowers the AHR and protects against inflammatory cell infiltration and mucus accumulation induced by house dust mite in both Zpbp2 KO and WT A/J mice. LAU-7b prevents Ormdl3 overexpression in WT allergic mice and corrects the aberrant levels of very-long-chain and long-chain ceramides in both WT and Zpbp2 KO allergic mice.
The research presented in this manuscript was funded by Canadian Institute of Health Research (CIHR) grant entitled Genetic and epigenetic control of ZPBP2/ORMDL3/GSDMB and susceptibility to asthma, to D.R. and A.K.N. and the CIHR grant to D.R. entitled Genetic dissection of airway hyperresponsiveness and susceptibility to allergic asthma. The Fenretinide clinical capsules were provided free of charge by Laurent Pharmaceuticals Inc., a company at which D.R. is a shareholder. These studies were supported by a grant from European Structural and Investment Funds Operational Program Research entitled Molecular, cellular and clinical approach to healthy ageing grant ENOCH (registration number: CZ.02.1.01/0.0/0.0/16_019/ 0000868) to M.H., D.R., and J.B.D.S. and by a grant from Czech Research Council 16-32302A to M.H.

https://doi.org/10.1124/jpet.119.263715.

S This article has supplemental material available at jpet.aspetjournals.org.

\section{Introduction}

Allergic asthma causes global health burdens because it affects millions of people worldwide. Being a complex disease, in addition to environmental exposures, several genetically controlled factors greatly influence the predisposition and severity of allergic asthma (Burbank et al., 2017). Genomewide association studies have highlighted the $17 \mathrm{q} 21$ locus, which contains several susceptibility genes, including zona pellucida binding protein 2 (ZPBP2) and ORMDL sphingolipid biosynthesis regulator 3 (ORMDL3) on human chromosome 17 and their orthologs Zpbp2 and Ormdl3 on mouse chromosome 11, as a well established susceptible locus for

ABBREVIATIONS: AA, arachidonic acid; AHR, airway hyper-responsiveness; CF, cystic fibrosis; DHA, docosahexaenoic acid; HDM, house dust mite; II-4, Interleukin-4; KO, knockout; LCC, long-chain ceramide; MCh, methacholine; MD, malondialdehyde; NT, nitrotyrosine; Ormdl3, ORMDL sphingolipid biosynthesis regulator 3; PAS, periodic acid-Schiff; Penh, enhanced pause; VLCC, very-long-chain ceramide; WBP, whole-body plethysmography; WT, wild-type; Zpbp2, zona pellucida binding protein 2. 
airway hyper-responsiveness (AHR) (Moffatt et al., 2007). The importance of $17 \mathrm{q} 21$ locus was evaluated in an ethnically diverse population, but differences based on ethnicity were not found (Kothari et al., 2018).

ZPBP2 localizes to the sperm acrosome, facilitating the binding of the spermatozoa to the oocyte's zona pellucida, so it is highly expressed in testes in both mice and humans (Torabi et al., 2017). ZPBP2 expression has also been detected in lower levels in human somatic airway epithelial cells (Moussette et al., 2017). Recently, our group (Kanagaratham et al., 2018) and Miller et al. (2018) have published that $Z p b p 2$ gene deletion attenuated AHR in C57BL/6 mice; nevertheless, the functional impact of $Z p b p 2$ on allergic asthma remains undiscovered. Ormdl3, which is involved in modulating the biosynthesis of ceramides, has been mapped downstream of $Z p b p 2$, and it was recently shown that the deletion of $Z p b p 2$ in C57BL/6 mice led to a decrease in the level of Ormdl3 expression (Miller et al., 2018). Compared with ZPBP2, more studies have been published pertinent to ORMDL3 and allergic asthma. It has been shown that increased expression of Ormdl3 positively correlates with airway hyper-reactivity (Davis et al., 2018). Transcriptomic and metabolomic analyses have linked the forced expiration volume in the first second / forced vital capacity (FEV1/FVC) ratio with ORMDL3 and dysregulated lipid metabolism in a study of asthmatic children (Kelly et al., 2018).

Moreover, elevated levels of ORMDL3 inhibit sphingolipid biosynthesis and result in inhibition of long-chain ceramides (LCCs; C16:0) and four species of very-long-chain ceramides (VLCCs; C22:0, C24:0, C24:1, and C24:2) (Kiefer et al., 2019). Debeuf et al. (2019) reported that transgenic mice overexpressing Ormdl3 had significantly lower levels of VLCCs (C24:0 and C24:1); by contrast, loss of Ormdl3 in knockout (KO) mice resulted in elevated levels of C24:0 and C24:1. Besides allergic asthma, several studies showed that the ORMDL3 locus is also associated with other numerous pathologies (Das et al., 2017), e.g., allergic rhinitis (Tomita et al., 2013), type 1 diabetes (Saleh et al., 2011), primary biliary cirrhosis (Mells et al., 2011), rheumatoid arthritis (Kurreeman et al., 2012), ulcerative colitis (Anderson et al., 2011), Crohn disease (Franke et al., 2010), and ankylosing spondylitis (Laukens et al., 2010).

Fenretinide, a vitamin A derivative, corrects the aberrant inflammatory responses and improves lung functions in both cystic fibrosis (CF) (Guilbault et al., 2008, 2009, Youssef et al., 2016, 2020; Garić et al., 2017) and allergic asthma (Kanagaratham et al., 2014; Youssef et al., 2020). In CF, our laboratory has shown that Fenretinide improves lung function by elevating levels of VLCCs and lowering levels of LCCs (Garić et al., 2017). Recently, a novel clinical oral formulation of Fenretinide (LAU-7b) with excellent bioavailability was tested using daily single oral capsules $(100,200$, and $300 \mathrm{mg})$ in patients with $\mathrm{CF}$ in a phase Ib clinical trial study (NCT02141958). The results of a phase I clinical trial demonstrated very promising pharmacokinetics and pharmacodynamics of LAU-7b. Currently, LAU-7b is being tested in a phase II clinical trial in patients with CF (NCT03265288). However, LAU-7b capsules have not yet been tested in patients suffering from allergic asthma.

In our study, we developed KO mice for $Z p b p 2$ gene on the $\mathrm{A} / \mathrm{J}$ genetic background. Unlike $\mathrm{C} 57 \mathrm{BL} / 6$, the $\mathrm{A} / \mathrm{J}$ strain of mice is not only atopic but also expresses genes regulating
AHR. Allergic asthma developed in this strain of mice is characterized by a very strong inflammatory response, a significant increase in airway hyperplasia, and an intense AHR, which can be measured after exposure to methacholine (MCh). Therefore, we have used this strain of mice to investigate the protective effects of LAU- $7 \mathrm{~b}$ treatment $(10 \mathrm{mg} / \mathrm{kg}$ per day for 9 days) against HDM-induced asthma. We aimed to validate the importance of the $Z p b p 2$ gene in allergic asthma, so we hypothesized that A/J KO mice with the Zpbp2 gene deletion would be associated with decreased AHR measured after exposure to MCh. Because the Ormdl3 gene product regulates the biosynthesis of ceramides, we expected that changes in Ormdl3 gene expression will correlate with changes in the distribution of specific species of ceramides. We hypothesized that the allergic asthma-induced augmentation of Ormdl3 can be prevented by treatment with LAU-7b in the A/J hyperresponsive strain of mice. Furthermore, we hypothesized that there is an association between the LAU-7b-induced improvement in lung physiology and the normalization of relative abundance of VLCCs and LCCs in the lungs.

\section{Materials and Methods}

Animal Model. Heterozygous B6.127S7-Zpbp $2^{t m 1 Z u k} / \mathrm{J}$ mice (Lin et al., 2007), which carry a deletion of $Z p b p 2$ exons 1-3, were purchased from the Jackson Laboratory (Bar Harbor, ME) and backcrossed for more than 10 generations (N10) to A/J inbred mice purchased from Jackson Laboratory. N10 Zpbp2 KO mice were intercrossed to generate homozygous KO A.127S7-Zpbp2 $2^{t m 1 Z u k}$ mice, referred to from this point on as KO. All experimental procedures were approved by the Animal Care Committee of McGill University Health Center, Montreal, QC, Canada. More information is provided in the Supplemental Material.

Mouse Groups and Batches. After sensitization (more information is provided in the Supplemental Material), the mice were split into the following groups: allergic, PBS-treated, unchallenged mice (HDM-PBS-PBS); allergic, PBS-treated, challenged mice (HDM-PBSHDM); and allergic, LAU-7b-treated, challenged mice (HDM-LAU-7bHDM). For simplification, the three groups are presented as "PBS," "HDM," and "LAU-7b," respectively. Eight different experiment batches using Zpbp2 WT and KO mice on the A/J background ( $n=$ 217 ) were tested. The results collected from all eight experiments were pooled together to make the final graphs.

AHR Measurements. AHR was measured using a Buxco plethysmograph system, ventilators, and nebulizers (Harvard Apparatus, Holliston, MA) as previously described (Kanagaratham et al., 2014, 2018). For the noninvasive whole-body plethysmography (WBP), the baseline enhanced pause (Penh) values were measured without anesthesia at the age of 8 weeks before the first sensitization. The invasive AHR measurements were done when sacrificing the mice on the day of the harvest. A nebulizer was used in both noninvasive and invasive lung experiments to administer ascending doses of MCh (acetyl $\beta$-methyl choline, Cat: A2251; Sigma Aldrich, Saint Louis, MO).

Lung Histology Analysis. H\&E staining was used to assess lung tissue recruitment of different inflammatory cells around airways. Quantifying LAU-7b effects was done by counting the number of infiltrated inflammatory cells among at least four airways/mice, averaged, and normalized versus the perimeter of the airway basement membrane as previously described (Kanagaratham et al., 2014). Periodic acid-Schiff (PAS) stain was used to visualize goblet cell hyperplasia in the lungs. PAS-positive cells in the airways were counted and normalized by dividing the counts by the perimeter of the basement membrane. Per mouse, at least four airways were counted as previously described (Kanagaratham et al., 2014). Airway smooth 
muscle mass was stained using specific $\alpha$-smooth muscle actin antibody as previously described by our laboratory(Camateros et al., 2007). Masson trichrome stain was used to assess collagen production as previously described (Chen et al., 2015).

Lipids and Markers of Oxidation Analysis. Lipid analysis was done using $25 \mathrm{mg}$ of macerated lung tissue from each mouse. Classic isolation of lipids was done as previously described by Folch et al. (1957), and the levels of different lipid species were measured using high-performance liquid chromatography tandem mass spectrometry as previously described (Guilbault et al., 2008).

Gene Expression Measurements. RNA was extracted from snap-frozen lungs using the RNeasy Mini kit (Qiagen). In total, $500 \mathrm{ng}$ of RNA was reverse transcribed into cDNA for each analyzed sample using the QuantiTect Reverse Transcription kit (Qiagen). Levels of expression of Ormdl3 mRNA were measured by using the CFX384 Touch Real-Time PCR Detection System and SsoFast EvaGreen Supermix (BioRad). Primers were designed using the primer Basic Local Alignment Search Tool (BLAST) online software, National Center for Biotechnology Information (NCBI). More information is provided in the Supplemental Material.

IgE Measurements in Serum. Plasma IgE levels were measured using an ELISA IgE mouse kit (BD OptEIA Biosciences) following the manufacturer's instructions.

Statistical Analysis. Data were pulled out from at least three independent experiments for each analysis. Data were analyzed using GraphPad Prism 6 (version 6.01; GraphPad Software, Inc., San Diego, CA). An ANOVA test was used for analyzing the results of more than two groups, and a $t$ test was used for analyzing the results of two groups. $P$ values of less than 0.05 were considered statistically significant. The number of mice $(n)$ used for each analysis is written in each figure caption.

\section{Results}

Gene Expression and Basal Lung Function Analysis. We confirmed that, by removing exons $1-3$ of the $5^{\prime}$ region of $Z p b p 2$, the mRNA gene expression was abolished (Fig. 1, A and B) using lung tissue collected from the $\mathrm{A} / \mathrm{J} Z p b p 2 \mathrm{KO}$ mice we developed. It has been previously reported (Miller et al., 2018) that deletion of Zpbp2 has downregulated Ormdl3 expression in lung epithelial cell lines. Therefore, we wanted to evaluate whether Ormdl3 gene expression might be decreased in the lungs of $Z p b p 2 \mathrm{KO} \mathrm{A} / \mathrm{J}$ mice. In WT control mice, PBS-challenged mice demonstrated very low gene expression of Ormal3 compared with the gene expression of HDMchallenged mice (Fig. 1, A and B). Furthermore, in Zpbp2KO mice, Ormdl3 gene expression increase even after HDM challenges was also abolished (half-fold expression in $Z p b p 2$ KO mice compared with 2- to 4-fold expression in WT mice) (Fig. 1, A and B). Then, we treated the WT mice with LAU-7b, and we measured $Z p b p 2$ and Ormdl3 gene expression. Our data show that LAU-7b treatment significantly decreased the expression of Ormdl3 in both WT males and females compared with HDM-challenged and PBS-treated mice (Fig. 1D).

We also tested the gene expression of four of the T helper 2 (Th2) immune pathway-associted genes (Fig. 1, E-H): Interleukin-4; Il-4 (differentiates naive T cells; Th0 into Th2 cells (Steinke and Borish, 2001)), $I l-5$ (activates eosinophils (Farne et al., 2017)), Il-13 that regulates the production of IgE (Rael and Lockey, 2011), and C-C MotifChemokine Ligand 11; Ccl11, also known as Eotaxin-1 (enhances the infiltration of eosinophils into the airways (Williams, 2015)). Our results show that after HDM sensitization and challenge, the expression of Il-5, Il-13, and Eotaxin-1 was significantly elevated in
WT and $Z p b p 2 \mathrm{KO}$ mice; meanwhile, the expression of $I l-4$ was significantly elevated in WT but not Zpbp2 KO mice. LAU$7 \mathrm{~b}$-treated WT mice have significantly lower expression of $\mathrm{Il}$ 4, Il-5, and eotaxin-1 genes but not $\mathrm{Il}-13$ compared with littermate WT controls. Noninvasive WBP was used to measure baseline respiratory functions before starting HDM sensitization and without sacrificing the mice (Fig. 1I). At a MCh dose of $25 \mathrm{mg} / \mathrm{ml}$, both WT males and females have significantly higher lung Penh values than KO males and females, respectively.

Assessment of AHR and IgE Levels. The results of WBP were confirmed by classic invasive measurement of lung resistance on the day of harvest (Fig. 2, A-D). HDM challenge significantly increased the lung resistance values of all mouse groups (males, females, WTs, and KOs) at MCh doses of 50 and $100 \mathrm{mg} / \mathrm{ml}$ compared with PBS-challenged mice. Consistent with the previously published data (Card et al., 2006; Antunes et al., 2010), our data demonstrate that WT males have higher lung resistance than WT females; similarly, KO males have higher lung resistance than $\mathrm{KO}$ females (Fig. 2, A and B). Lung responsiveness to nebulized MCh revealed that WT (male and female) and $\mathrm{KO}$ (male) mice gavaged with $10 \mathrm{mg} / \mathrm{kg}$ LAU-7b for 9 days had significantly lower lung resistance than PBStreated mice (Fig. 2, C and D). Allergic KO females display a very low increase in lung resistance after HDM challenge (nonsignificant from PBS), and the treatment does not improve the lung physiology any further, which under these circumstances, is not surprising in this group of mice.

The protective effects of LAU-7b against increased airway hyper-responsiveness after allergen challenge prompted us to evaluate its potential against IgE production, which is also associated with allergic asthma. After HDM challenge, the titer of IgE was increased by 4- to 10-fold in WT and KO mice (Fig. 2E). IgE measurements show that WT and KO male and female groups were not statistically significant from one another. Nonetheless, LAU-7b treatment did not lower the levels of IgE caused by HDM challenge in WT and KO male and female mice compared with PBS-treated and HDMchallenged mice.

Evaluation of Inflammatory Cell Infiltration, Mucus Production, Smooth Muscle Mass, and Collagen Production in the Airways. To visualize and quantify the inflammatory cells' infiltration into the airways after HDM challenge, we used H\&E staining (Fig. 3). The lung sections of both WT and KO mice showed significant incoming inflammatory cells after HDM challenge compared with PBSchallenged mice (Fig. 3, A-L). We also quantified our H\&E staining results by counting and normalizing the recruited cells around the lung airways (Fig. 3M). Our data demonstrated that there was equally strong inflammatory cell infiltration into the airways of both WT and KO male and female mice after HDM allergen challenge, even though KO mice had displayed much less lung resistance than WT mice. As shown in the representative pictures from each mouse group, LAU-7b treatment significantly lowered the recruitment of inflammatory cells in both WT and KO male and female mice.

Mucus hypersecretion, and the subsequent plugging of the airways, has long been recognized as a common phenotype of allergic asthma. The mucus production was not investigated before in the $Z p b p 2 \mathrm{KO}$ mouse model, so in our study, we wanted to examine whether the deletion of this gene would 

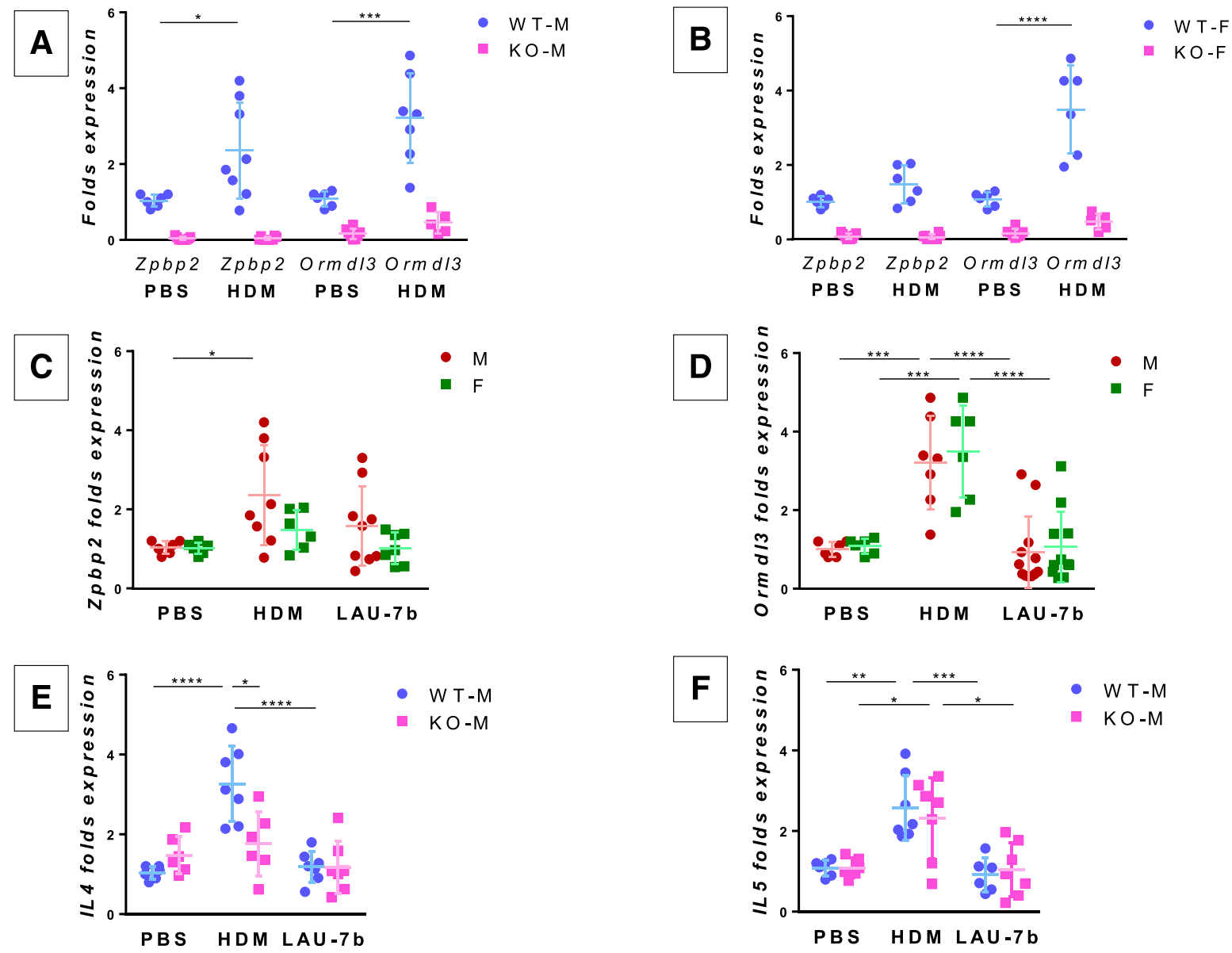

- $W T-M$
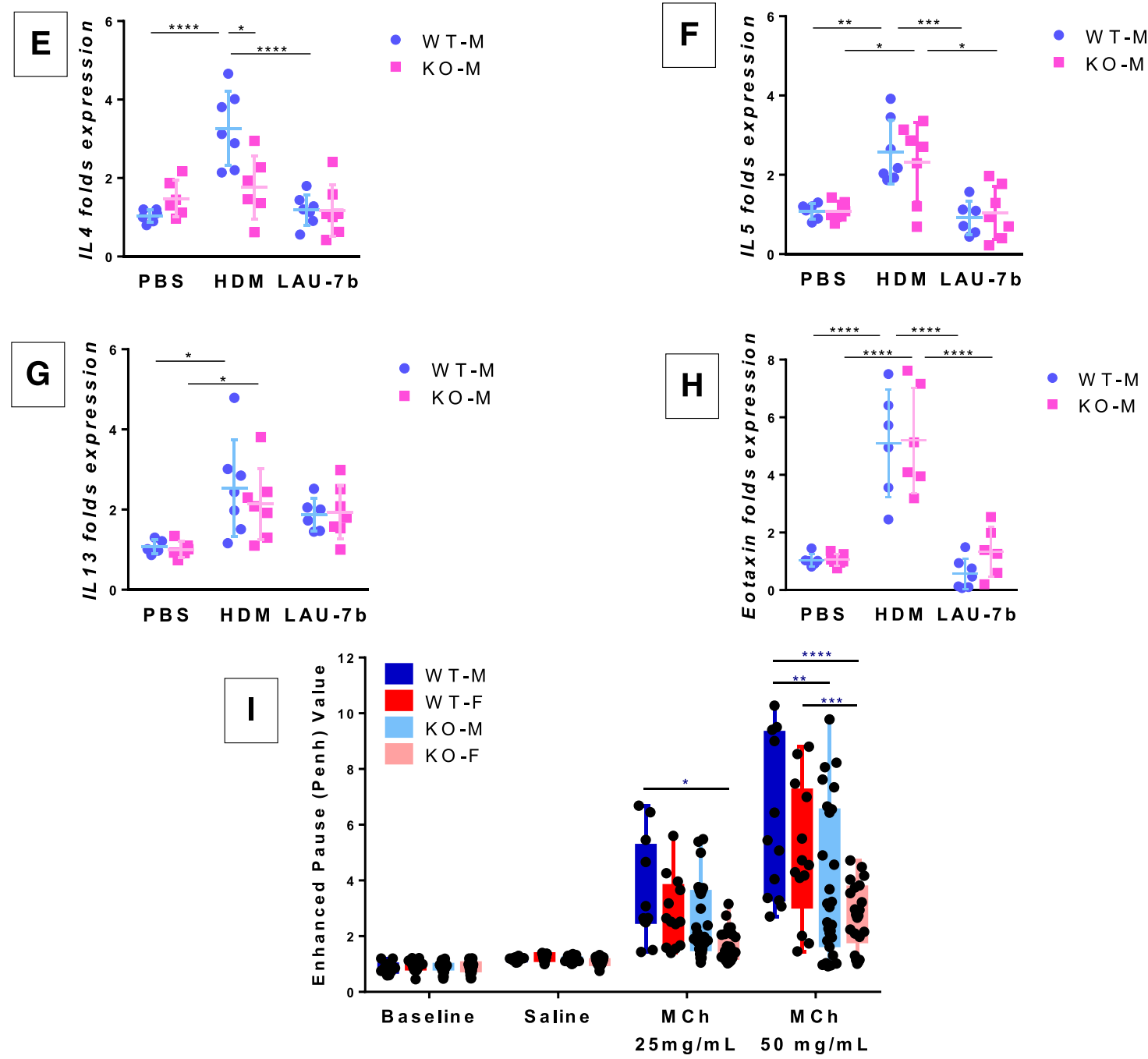

Fig. 1. Zpbp2 and Ormdl3 gene expression and basal lung function in WT vs. Zpbp2 KO mice fold-change expression of Zpbp2 and Ormdl3 genes quantitated using qPCR for WT and Zpbp2 KO (A) male mice and (B) female mice. Fold-change expression of (C) Zpbp2 and (D) Ormdl3 genes quantitated using qPCR for LAU-7b-treated WT male mice and female mice. LAU-7b treatment and fold-change expression of (E) $I l-4$, (F) $I l-5$, (G) $I l-13$, and $(\mathrm{H})$ Eotaxin-1 genes in Zpbp2 KO and WT mice. All mice were sensitized with HDM and challenged with either PBS or HDM. Treatment group is marked as LAU-7b. (I) Noninvasive WBP for WT and Zpbp2 KO mice. Penh values were measured in response to increasing doses of inhaled MCh in 
result in any effects that could be visualized by the PAS stain (Fig. 4) and corrected with LAU-7b treatment. Our lung sections of both WT and KO mice markedly show mucus production by goblet cells after HDM challenge compared with PBS-challenged mice (Fig. 4, A-L). No significant differences between WT and KO male or female mice were observed. LAU$7 \mathrm{~b}$ treatment significantly decreased the production of mucus by goblet cells in both WT and KO male and female mice (Fig. 4M).

As remodeling of the airways is an important aspect in the context of human asthma, we wanted to assess the smooth muscle mass and collagen production in our mouse model and evaluate the effect of treatment with LAU-7b. Our data (Supplemental Fig. 1) show that after HDM challenge, there is a slight increase in the mass of the smooth muscles of the mouse airways. Similarly, challenge with HDM results in the production of collagen (Supplemental Fig. 2), which was evident in the airways. LAU-7b treatment lowered both the mass of the smooth muscles and the collagen production in our $\mathrm{KO}$ and WT A/J mice.

Analysis of Ceramides, Fatty Acids, and Markers of Oxidation. We evaluated the levels of malondialdehyde (MD), a marker of lipid oxidation, and nitrotyrosine (NT), a marker of protein oxidation, in our mice because they are markers of cellular stress and damage, which happen after allergen challenge. After HDM challenge, both MD and NT significantly increased in male and female $\mathrm{KO}$ and WT mice (Fig. 5, A and B). No significant differences were observed between males and females or KO and WT mice. LAU-7b significantly normalized the levels of MD and NT in the HDMchallenged and treated mice compared with HDM-challenged and untreated mice.

Furthermore, fatty acid analysis (Fig. 5C) reveals significant elevation in the arachidonic acid/docosahexaenoic acid ratio (AA/DHA ratio) after HDM challenge, which is typically associated with inflammation. Nevertheless, this ratio is significantly lowered after treatment with LAU-7b in HDMsensitized and -challenged mice compared with the untreated HDM-sensitized and -challenged mice. Likewise, no significant differences in the AA/DHA ratio were observed between males and females or KO and WT mice.

The lipidomic analysis of the lungs (Fig. 5, D and E; Supplemental Fig. 3) revealed that the relative levels of VLCCs (C22:0, C22:1, C24:0, C24:1, C26:0, C26:1) were diminished in HDM-challenged KO mice (females $=24.07 \%$, lower than males $=34.45 \%$ ) compared with PBS-challenged KO mice (females $=34.44 \%$, lower than males $=37.93 \%$ ). LAU7b-treated KO mice displayed higher levels of VLCCs compared with untreated KO mice in both males and females: $40.70 \%$ and $40.24 \%$, respectively. In KO mice, treatment with LAU-7b resulted in significantly elevated levels of C24:0, C24: 1, C26:0, and C26:1 in males and C24:0 in females. Similarly, the treatment with LAU-7b resulted in significantly reduced levels of LCCs: C14:0 and C18:0 in KO males and C14:0, C16:0, and C18:0 in KO females.

Moreover, compared with KO mice, the assessment of VLCCs demonstrated that WT male and female mice have higher levels of VLCCs (Supplemental Fig. 3). Total VLCC levels in PBS-challenged WT mice demonstrated a percentage of $41.85 \%$ in males and $43.07 \%$ in females. After challenge with HDM, the total VLCC levels were reduced to $39.92 \%$ in males and $31.06 \%$ in females. As shown here, the VLCC values obtained from male and female $\mathrm{KO}$ mice were lower than the values obtained for male and female WT mice for both PBSand HDM-challenged groups. However, as observed in KO mice, LAU-7b treatment restored the levels of VLCCs to those typically observed in WT male and female mice-up to $42.43 \%$ and $47.89 \%$, respectively-which is even higher than the levels of VLCCs detected before HDM challenge (in PBS mouse groups).

\section{Discussion}

The importance of the 17q21 locus, containing genes such as $O R M D L 3$ and $Z P B P 2$, was replicated in several studies (Moffatt et al., 2007; Karunas et al., 2011; Torgerson et al., 2011; Wan et al., 2012). Our main objective was 3-fold. First, we aimed to evaluate the effect of the $Z p b p 2$ gene deletion on lung physiology. Secondly, we aimed to investigate the effects of LAU-7b treatment against HDM-induced allergic asthma in $Z p b p 2 \mathrm{KO}$ and WT A/J mice. Our third objective was to test the expression of $Z p b p 2$ and $O r m d l 3$ genes after LAU-7b treatment to evaluate whether there might be an association between the modulation of the expression of these genes and the changes in the regulation of relative ratios of VLCCs and LCCs.

We have previously published (Kanagaratham et al., 2018) that the deletion of the $Z p b p 2$ gene in C57BL/ 6 mice resulted in a reduction of AHR in females but not males on baseline levels. However, the deletion of the $Z p b p 2$ gene in our previous study (Kanagaratham et al., 2018) did not significantly affect the AHR of male or female mice after ovalbumin sensitization and challenge, perhaps because the C57BL/6 strain of mice is genetically resistant to developing allergic asthma. Miller et al. (2018) similarly reported that Zpbp2 KO C57BL/6 male and female mice challenged with HDM had significantly reduced AHR compared with WT controls. Our results here demonstrated that deletion of the $Z p b p 2$ gene on the A/J background has resulted in a significant reduction of baseline Penh values in male and female mice (Fig. 1I) compared with WT controls. Similarly, we observed a significant reduction in AHR (shown by the lung resistance values, Fig. 2, A and B) of $Z p b p 2 \mathrm{KO}$ male and female mice compared with WT mice. By using an $\mathrm{A} / \mathrm{J}$ hyper-responsive strain of mice and inducing allergic asthma by HDM, we have obtained a good segregation of different mouse groups (KO and WT, males and females) in terms of AHR. Our data demonstrate for the first time that in the genetically very susceptible (high AHR) and atopic mice, this gene plays an important role in the control of lung physiology in allergic asthma.

HDM sensitization and challenge significantly enhanced the recruitment of inflammatory cells into the lungs (Fig. 3), caused hyperplasia of the lining of the airways (Fig. 3), and increased the production of mucus (Fig. 4) in Zpbp2 $\mathrm{KO}$ and

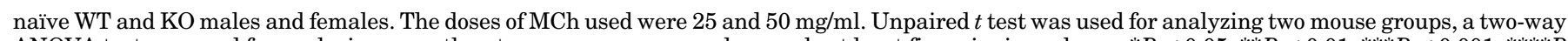

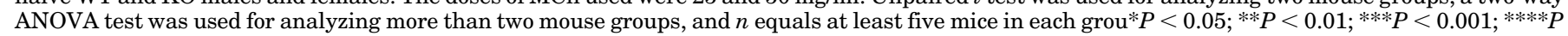
$<0.0001$. F, female; M, male; qPCR, quantitative polymerase chain reaction. 

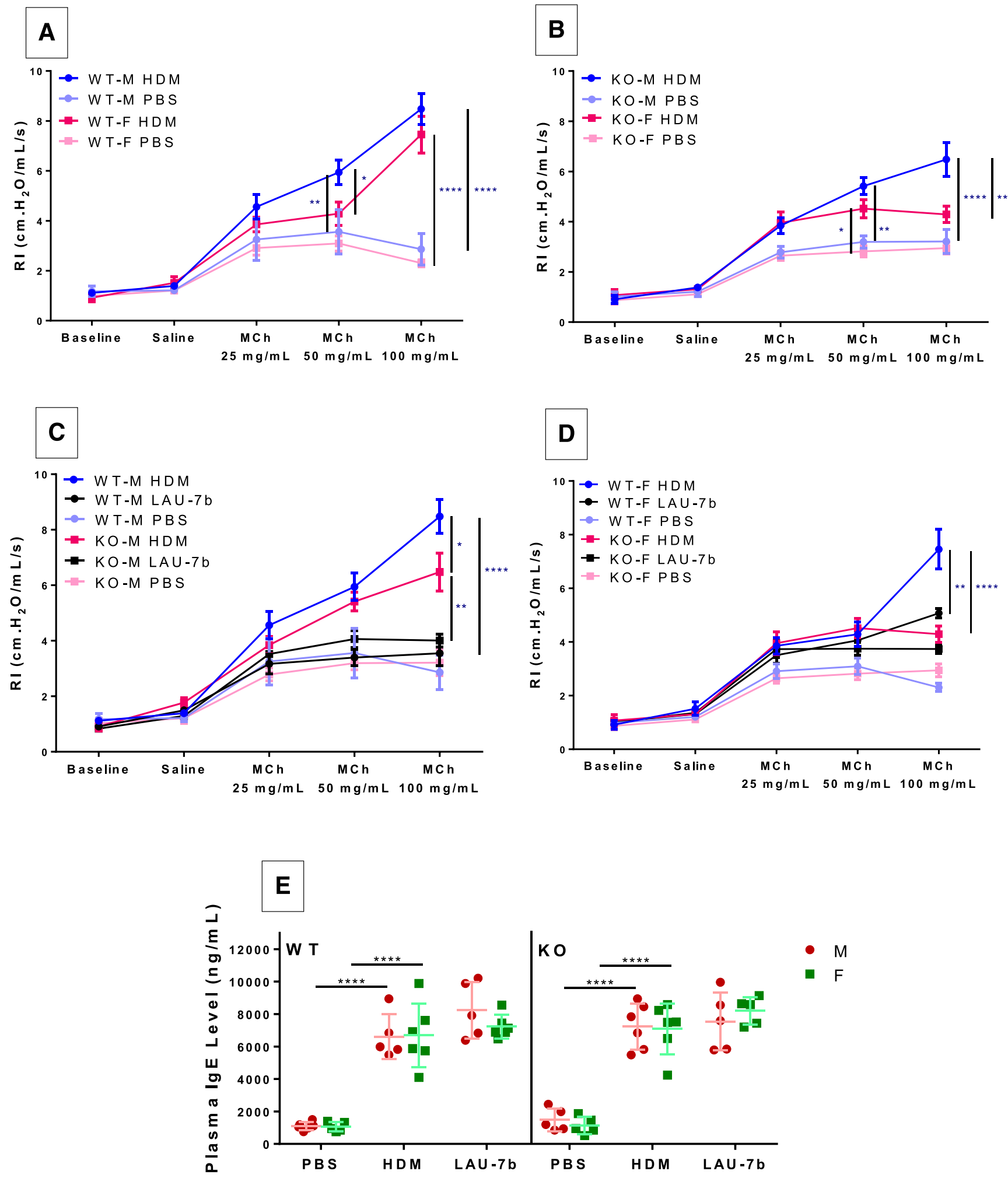

Fig. 2. Classic invasive measurements of lung resistance and measurements of IgE levels of $Z p b p 2 \mathrm{KO}$ and WT mice. Classic invasive measurements of lung resistance in (A) WT and (B) Zpbp2 KO mice. All mice were sensitized with HDM and challenged with either PBS or HDM. Treatment group is marked as LAU-7b. The doses of MCh used were 25,50 , and $100 \mathrm{mg} / \mathrm{ml}$. LAU-7b treatment and airway hyper-responsiveness in MCh nebulized male (C) and female (D) mice. Two-way ANOVA, $n$ equals at least 11 mice in each group. (E) Treatment with LAU-7b did not affect the levels of IgE in $Z p b p 2 \mathrm{KO}$ and WT mice. Two-way ANOVA, $n$ equals at least five mice for each group. $* P<0.05$; $* *<0.01$; $* * * *<0.0001$. F, female; M, male. RI: inspiratory airway resistance. 

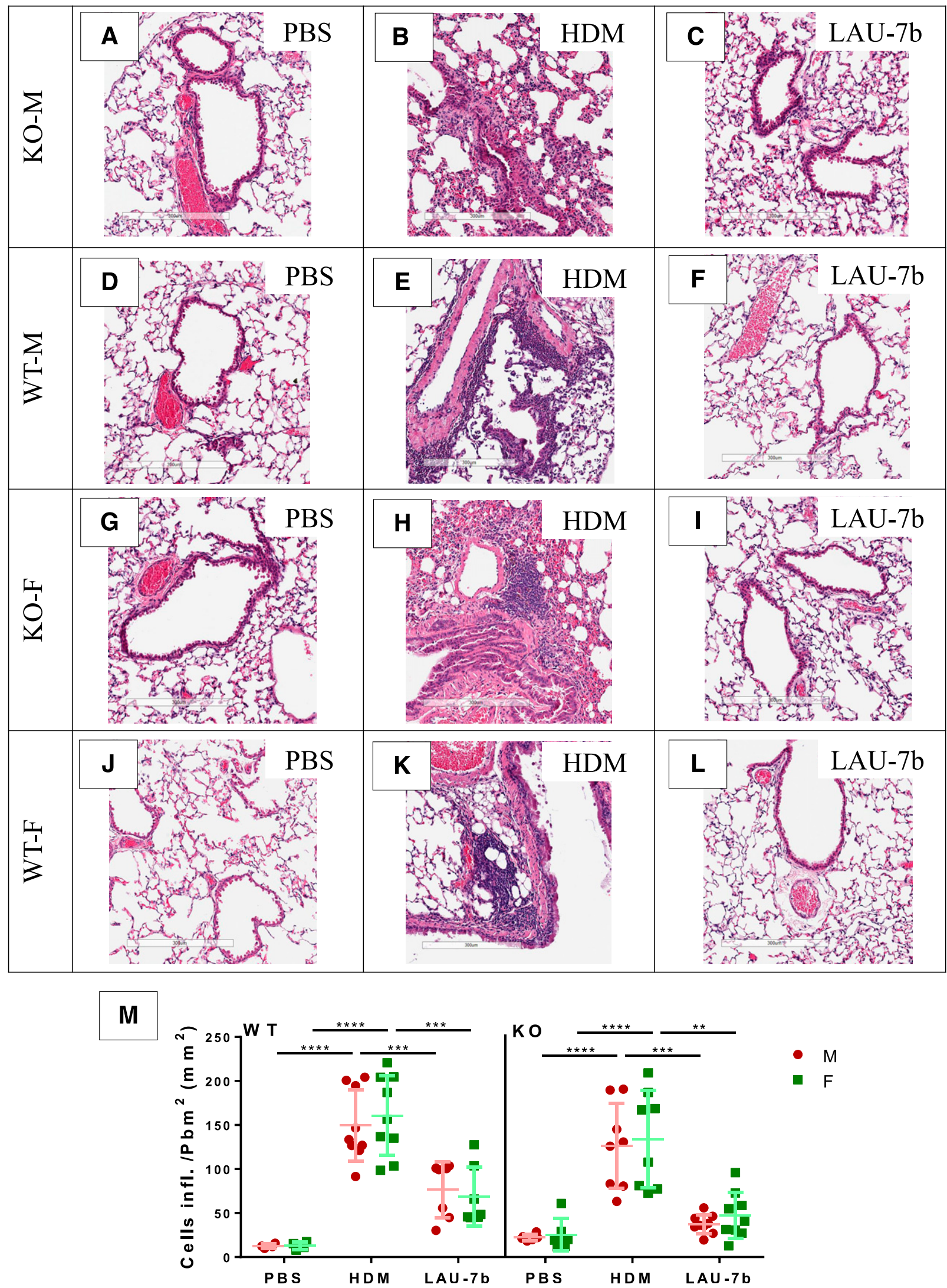

Fig. 3. Infiltration of airways by inflammatory cells in untreated and LAU-7b-treated mice; H\&E staining. All mice were sensitized with HDM and challenged with either PBS or HDM. Treatment group is marked as LAU-7b. (A-C) Zpbp2 KO males, (D-F) WT males, (G-I) Zpbp2 KO females, and (J-L) WT females. (M) LAU-7b-treated mice have significantly lower lung cell infiltration compared with the lungs of untreated mice. Quantification was done by counting the number of inflammatory cells around each airway and normalizing it by division over the square of the perimeter "in millimeters" of the airway basement membrane. Measurements were done using at least four different airways for each mouse from each group. $n$ equals at least seven mice for each group, two-way ANOVA. ${ }^{* *} P<0.01 ; * * * P<0.001 ; * * * * P<0.0001$. F, female; M, male. Scale bar $=300 \mu \mathrm{m}$. 

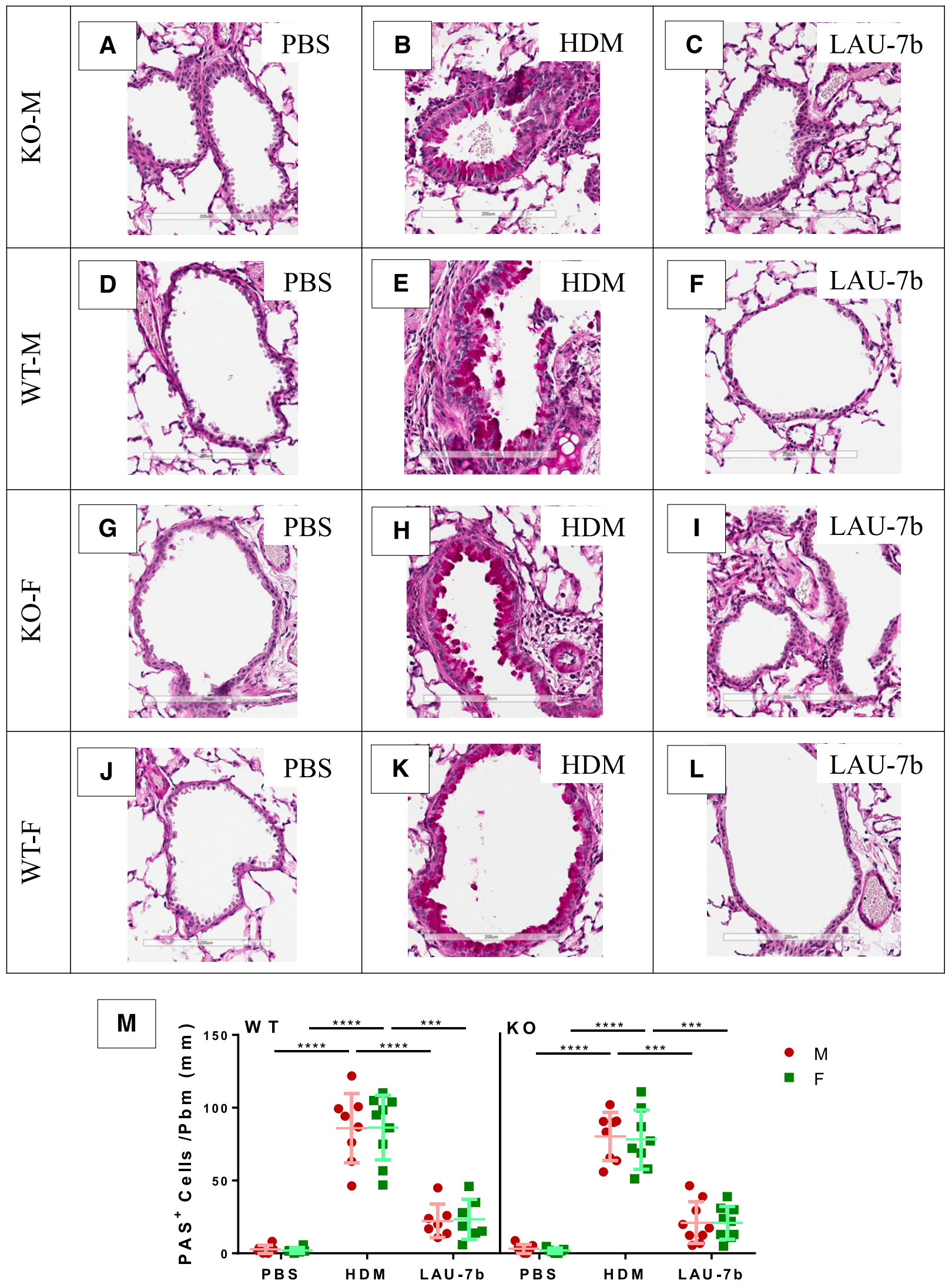

Fig. 4. Mucus production by goblet cells in untreated and LAU-7b-treated mice. PAS staining. All mice were sensitized with HDM and challenged with either PBS or HDM. Treatment group is marked as LAU-7b. (A-C) Zpbp2 KO males, (D-F) WT males, (G-I) Zpbp2 KO females, and (J-L) WT females. (M) LAU-7b-treated mice have significantly lower production of mucus by goblet cells compared with untreated mice. Quantification was done by counting the number of PAS-positive cells around each airway and normalizing it by division over the perimeter "in millimeters" of the airway basement membrane. Measurements were done using at least four different airways for each mouse from each group. $n$ equals at least seven mice for each group, two-way ANOVA. $* * * P<0.001 ; * * * * P<0.0001$. F, female; M, male. Scale bar $=200 \mu \mathrm{m}$. 

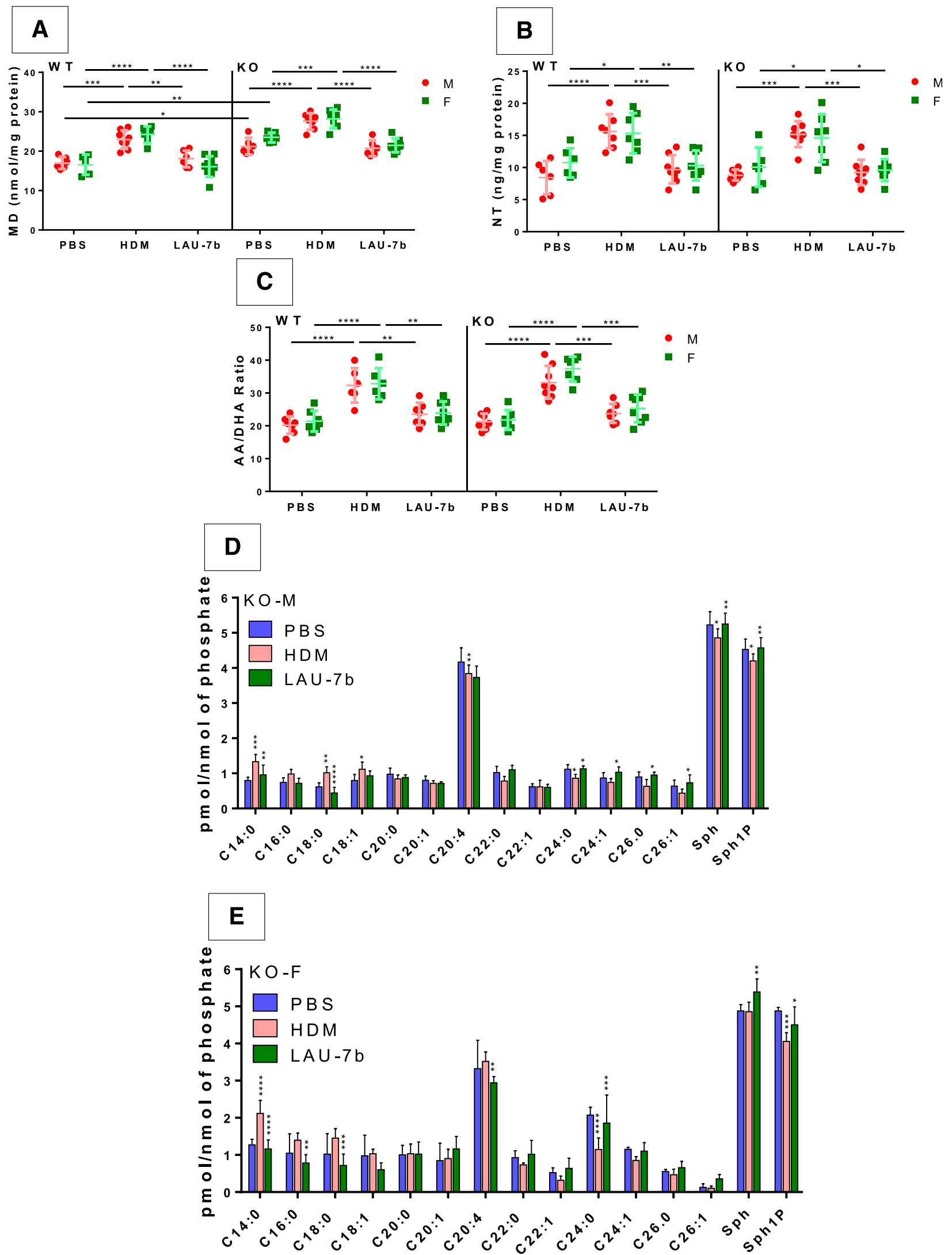

Fig. 5. LAU-7b treatment corrected the aberrant levels of markers of oxidation, fatty acids, and sphingolipids in $Z p b p 2 \mathrm{KO}$ and WT mice. Analysis of (A) $\mathrm{MD}$, marker of lipid oxidation; (B) NT, marker of protein oxidation; and (C) AA/DHA ratio. Analysis of sphingolipid species in (D) $Z p b p 2 \mathrm{KO}$ male and (E) Zpbp2 KO female mice. All mice were sensitized with HDM and challenged with either PBS or HDM. Treatment group is marked as LAU-7b. Two-way ANOVA, $n$ equals at least six mice for each group. ${ }^{*} P<0.05$; $* * P 0.01 ; * * * P<0.001 ; * * * * P<0.0001$. F, female; M, male. Sph: sphingosine and Sph1P: sphingosine-1-phosphate species. 
WT mice. Interestingly, the ablation of the Zpbp2 gene, although it affected the lung resistance, did not result in the modulation of inflammatory response after allergen challenge. Although $Z p b p 2 \mathrm{KO}$ mice have reduced AHR after HDM challenge, the inflammatory response demonstrated by the recruitment of cells into the airways and excessive mucus production was still induced. Altogether, in WT and Zpbp2 KO mice (males and females), LAU-7b treatment had resulted in significantly lower cell infiltration, no hyperplasia, and inhibited mucus production compared with PBS-treated mice of the same genotype and sex.

To determine whether $Z p b p 2$ deficiency influenced the levels of IgE, a common marker of allergy, we quantified IgE in plasma, which was statistically elevated after HDM challenge in $\mathrm{KO}$ males and females to similar levels present in WT mice (Fig. 2E). Although LAU-7b treatment did not correct elevated plasma IgE levels either in $\mathrm{KO}$ males or in females [findings were similar to our previously published data (Kanagaratham et al., 2014, 2018)], it was still able to control the inflammatory response to the allergen in the lungs and normalize lung physiology of the allergen-sensitized and -challenged animals. These findings suggest that, even in the most severely affected patients who are not responding to either steroids or to anti-IgE therapy anymore, this type of treatment might still be effective.

Ormdl3 plays a significant role in allergic asthma (Paulenda and Draber, 2016). In yeast, ORM proteins negatively regulate sphingolipid synthesis by forming a conserved complex with the serine palmitoyl transferase enzyme, thus inhibiting the first rate-limiting step of de novo ceramide, and all other sphingolipids', biosynthesis (Breslow et al., 2010). In a mouse study, myriocin, an inhibitor of the serine palmitoyl transferase enzyme, reduced the de novo sphingolipid synthesis and increased bronchial reactivity (Worgall et al., 2013). Likewise, increased airway responsiveness and airway remodeling has been reported in C57BL/6 transgenic mice overexpressing Ormdl3 after ovalbumin sensitization and challenge (Miller et al., 2014). Similarly, C57BL/6 mice lacking Ormdl3 were protected from developing AHR and airway eosinophilia induced by Alternaria alternata (Löser et al., 2017).

Further, the expression of ORMDL3 is not independent of the expression of other genes present in the 17q21 chromosomal locus. It has been reported that ZPBP2 and ORMDL3 genes are coregulated together as closely associated cishaplotype elements (Verlaan et al., 2009). In our study, we report that there is significantly lower Ormdl3 gene expression in $\mathrm{A} / \mathrm{J} Z p b p 2 \mathrm{KO}$ mice than in the littermate WT controls (Fig. 1D). The finding that knocking out the Zpbp2 gene in A/J mice has markedly reduced AHR can be, at least partially, attributed to the downregulation of two hyperresponsiveness susceptibility genes (Ormdl3 and $\mathrm{Zpbp2}$ ) since this strain of mice expresses genes that make them both atopic (loci on chromosome 4) and display increased AHR (loci on chromosome 12).

It has been reported that elevated levels of ORMDL3 inhibit sphingolipid biosynthesis, resulting in inhibition of both LCCs (C16:0) and four species of VLCCs (C22:0, C24:0, C24:1, and C24:2) (Kiefer et al., 2019). By contrast, Zhang et al. (2019) had reported that ORMDL3 gene silencing in A549 and normal human bronchial epithelial (NHBE) cell lines resulted in a marked increase in the levels of C24:0, C24:1, C26:1, and sphingosine-1-phosphate species. Recently, Debeuf et al. (2019) reported that transgenic mice overexpressing Ormdl3 had significantly reduced levels of C24:0 and C24:1. By contrast, loss of Ormdl3 in mice resulted in elevated levels of C24:0 and C24:1. Interestingly, our results demonstrated that LAU-7b treatment had lowered the expression of Ormdl3 (Fig. 1D) and protected the sensitized and challenged WT mice against allergic asthma. It is well established that Ormdl3 overexpression inhibits de novo sphingolipid biosynthesis; therefore, the LAU-7b-induced downregulation of Ormdl3 gene expression in WT mice may explain the reason why we observed an increase in the levels of VLCCs in LAU7b-treated mice (Fig. 5). Although Zpbp2 KO mice have lower AHR after HDM sensitization and challenge, the inflammatory reaction after allergen exposure still occurred to its full capacity. It is intriguing that LAU-7b treatment was able to protect the $Z p b p 2 \mathrm{KO}$ mice against the inflammatory reactions after HDM sensitization and challenge. This effect cannot be explained by the ability of LAU-7b to modulate Ormdl3 expression, since our Zpbp2 KO mouse model has ablated expression of $Z p b p 2$ and reduced expression of Ormdl3.

We tested four genes (Il-4, Il-5, Il-13, and Eotaxin-1) that are overexpressed by $\mathrm{T}$ helper type 2 cells in allergic asthma (Fig. 1, E-H). After challenge with HDM, both Zpbp2 KO and WT mice had significantly increased expression of $I l-5, I l-13$, and Eotaxin-1 genes compared with PBS-challenged control mice (Fig. 1, E-H). However, for these three genes, no significant differences were observed between Zpbp2 KO and WT mice. These findings coincide with nonsignificant differences between the two mouse strains in terms of elevated IgE levels (Fig. 2E) and an increased number of inflammatory cells recruited into the airways (Fig. 3) after allergen challenge. In our Zpbp2 $\mathrm{KO}$ mouse model, the expression of Il-4 was not elevated significantly after HDM challenge compared with PBS KO controls, unlike HDM-challenged WT mice, which show significant elevation of $I l-4$ levels. Miller et al. (2018) reported a lack of significant increase in IL-13 levels in $Z p b p 2 \mathrm{KO}$ mice after HDM challenge compared with $Z p b p 2$ KO controls. The results of the studies reported by Debeuf et al. (2019) also demonstrated the lack of a significant increase in the levels of IL-13 or IL-5 after HDM challenge when the mice were either overexpressing, or had displayed the loss of, Ormdl3. Levels of IL-4 were not reported in these two studies cited above. Taken together, there is no evidence linking the alteration of $\mathrm{Zpbp} 2$ or Ormdl3 genes with modulation of Il-4, Il-5, Il-13, or Eotaxin-1 expression.

Our studies clearly demonstrate that treatment of allergensensitized and allergen-challenged mice with LAU-7b had lower expression of $I l-4$ (WT mice), Il-5, and Eotaxin-1 (WT and KO mice) compared with PBS-treated mice, which is consistent with the previously reported effect on extracellular signal-regulated protein kinases 1 and 2 (ERK1/2) phosphorylation (Lachance et al., 2013). IL-13 is a key regulatory cytokine in the production of IgE (Rael and Lockey, 2011). Interestingly, both $Z p b p 2 \mathrm{KO}$ and WT mice treated with LAU$7 \mathrm{~b}$ did not show significant reduction of $\mathrm{Il}-13$ expression, which may explain why IgE levels remained elevated in both groups. Interestingly, inhibition of IgE levels was not essential for improving lung physiology in treated animals.

Overall, the preclinical data presented here for Fenretinide, the active pharmaceutical ingredient of LAU-7b capsules, 
provide robust grounds that justify testing the efficacy of this dosage form in a clinical trial, especially among asthmatics whose severe asthma is no longer treatable using the currently available therapies.

\section{Conclusions}

LAU-7b treatment in a dose of $10 \mathrm{mg} / \mathrm{kg}$ per day for 9 days protects both $Z p b p 2 \mathrm{KO}$ mice, which have significantly reduced Ormdl3 expression, and their WT littermate controls from HDM-induced allergic asthma. Interestingly, in WT mice, LAU-7b had significantly lowered the expression of Ormdl3, and hence, it can be effective in allergic asthma treatment by elevating the levels of VLCCs and decreasing the levels of LCCs.

\section{Authorship Contributions}

Participated in research design: Youssef, Naumova, Radzioch.

Conducted experiments (animal experiments, whole-body plethysmography, airway resistance measurements, histology work and IgE measurement): Youssef. (RNA expression analysis): Youssef, Shah, Dumut. (Measurements of lipids, ceramides and markers of oxidative stress): De Sanctis, Hajduch.

Performed data analysis: Youssef.

Wrote or contributed to the writing of the manuscript: Youssef, De Sanctis, Shah, Dumut, Hajduch, Naumova, Radzioch.

\section{References}

Anderson CA, Boucher G, Lees CW, Franke A, D'Amato M, Taylor KD, Lee JC, Goyette P, Imielinski M, Latiano A, et al. (2011) Meta-analysis identifies 29 additional ulcerative colitis risk loci, increasing the number of confirmed associations to 47. Nat Genet 43:246-252.

Antunes MA, Abreu SC, Silva AL, Parra-Cuentas ER, Ab'Saber AM, Capelozzi VL, Ferreira TPT, Martins MA, Silva PMR, and Rocco PRM (2010) Sex-specific lung remodeling and inflammation changes in experimental allergic asthma. $J$ Appl Physiol (1985) 109:855-863.

Breslow DK, Collins SR, Bodenmiller B, Aebersold R, Simons K, Shevchenko A Ejsing CS, and Weissman JS (2010) Orm family proteins mediate sphingolipid homeostasis. Nature 463:1048-1053.

Burbank AJ, Sood AK, Kesic MJ, Peden DB, and Hernandez ML (2017) Environmental determinants of allergy and asthma in early life. $J$ Allergy Clin Immunol 140:1-12.

Camateros P, Tamaoka M, Hassan M, Marino R, Moisan J, Marion D, Guiot M-C, Martin JG, and Radzioch D (2007) Chronic asthma-induced airway remodeling is prevented by toll-like receptor-7/8 ligand S28463. Am J Respir Crit Care Med 175 1241-1249.

Card JW, Carey MA, Bradbury JA, DeGraff LM, Morgan DL, Moorman MP, Flake GP, and Zeldin DC (2006) Gender differences in murine airway responsiveness and lipopolysaccharide-induced inflammation. J Immunol 177:621-630.

Chen J, Zhou H, Wang J, Zhang B, Liu F, Huang J, Li J, Lin J, Bai J, and Liu R (2015) Therapeutic effects of resveratrol in a mouse model of HDM-induced allergic asthma. Int Immunopharmacol 25:43-48.

Das S, Miller M, and Broide DH (2017) Chromosome 17q21 genes ORMDL3 and GSDMB in asthma and immune diseases. Adv Immunol 135:1-52.

Davis D, Kannan M, and Wattenberg B (2018) Orm/ORMDL proteins: gate guardians and master regulators. Adv Biol Regul 70:3-18.

Debeuf N, Zhakupova A, Steiner R, Van Gassen S, Deswarte K, Fayazpour F, Van Moorleghem J, Vergote K, Pavie B, Lemeire K, et al. (2019) The ORMDL3 asthma susceptibility gene regulates systemic ceramide levels without altering key asthma features in mice. J Allergy Clin Immunol 144:1648-1659.e9.

Farne HA, Wilson A, Powell C, Bax L, and Milan SJ (2017) Anti-IL5 therapies for asthma. Cochrane Database Syst Rev 9:CD010834.

Folch J, Lees M, and Sloane Stanley GH (1957) A simple method for the isolation and purification of total lipides from animal tissues. $J$ Biol Chem 226:497-509.

Franke A, McGovern DPB, Barrett JC, Wang K, Radford-Smith GL, Ahmad T, Lees CW, Balschun T, Lee J, Roberts R, et al. (2010) Genome-wide meta-analysis increases to 71 the number of confirmed Crohn's disease susceptibility loci. Nat Genet 42:1118-1125.

Garić D, De Sanctis JB, Wojewodka G, Houle D, Cupri S, Abu-Arish A, Hanrahan JW, Hajduch M, Matouk E, and Radzioch D (2017) Fenretinide differentially modulates the levels of long- and very long-chain ceramides by downregulating Cers5 enzyme: evidence from bench to bedside. J Mol Med (Berl) 95:1053-1064.

Guilbault C, De Sanctis JB, Wojewodka G, Saeed Z, Lachance C, Skinner TAA Vilela RM, Kubow S, Lands LC, Hajduch M, et al. (2008) Fenretinide corrects newly found ceramide deficiency in cystic fibrosis. Am J Respir Cell Mol Biol 38: 47-56.

Guilbault C, Wojewodka G, Saeed Z, Hajduch M, Matouk E, De Sanctis JB, and Radzioch D (2009) Cystic fibrosis fatty acid imbalance is linked to ceramide deficiency and corrected by fenretinide. Am J Respir Cell Mol Biol 41:100-106.
Kanagaratham C, Chiwara V, Ho B, Moussette S, Youssef M, Venuto D, Jeannotte L, Bourque G, de Sanctis JB, Radzioch D, et al. (2018) Loss of the zona pellucidabinding protein 2 (Zpbp2) gene in mice impacts airway hypersensitivity and lung lipid metabolism in a sex-dependent fashion. Mamm Genome 29:281-298.

Kanagaratham C, Kalivodová A, Najdekr L, Friedecký D, Adam T, Hajduch M, De Sanctis JB, and Radzioch D (2014) Fenretinide prevents inflammation and airway hyperresponsiveness in a mouse model of allergic asthma. Am J Respir Cell Mol Biol 51:783-792.

Karunas AS, Iunusbaev BB, Fedorova IuIu, Gimalova GF, Ramazanova NN, Gur'eva LL, Mukhtarova LA, Zagidullin ShZ, Etkina EI, and Khusnutdinova EK (2011) [Genome-wide association study of bronchial asthma in the Volga-Ural region of Russia]. Mol Biol (Mosk) 45:992-1003.

Kelly RS, Chawes BL, Blighe K, Virkud YV, Croteau-Chonka DC, McGeachie MJ, Clish CB, Bullock K, Celedón JC, Weiss ST, et al. (2018) An integrative transcriptomic and metabolomic study of lung function in children with asthma. Chest 154:335-348.

Kiefer K, Casas J, García-López R, and Vicente R (2019) Ceramide imbalance and impaired TLR4-mediated autophagy in BMDM of an ORMDL3-overexpressing mouse model. Int J Mol Sci 20:E1391.

Kothari PH, Qiu W, Croteau-Chonka DC, Martinez FD, Liu AH, Lemanske RF Jr, Ober C, Krishnan JA, Nicolae DL, Barnes KC, et al. Asthma BioRepository for Integrative Genomic Exploration (Asthma BRIDGE) Consortium (2018) Role of local CpG DNA methylation in mediating the $17 \mathrm{q} 21$ asthma susceptibility gasdermin B (GSDMB)/ORMDL sphingolipid biosynthesis regulator 3 (ORMDL3) expression quantitative trait locus. J Allergy Clin Immunol 141:2282-2286.e6 DOI: 10.1016/j.jaci.2017.11.057.

Kurreeman FAS, Stahl EA, Okada Y, Liao K, Diogo D, Raychaudhuri S, Freudenberg J, Kochi Y, Patsopoulos NA, Gupta N, et al.; CLEAR investigators (2012) Use of a multiethnic approach to identify rheumatoid- arthritis-susceptibility loci, 1p36 and 17q12. Am J Hum Genet 90:524-532.

Lachance C, Wojewodka G, Skinner TAA, Guilbault C, De Sanctis JB, and Radzioch D (2013) Fenretinide corrects the imbalance between omega- 6 to omega-3 polyunsaturated fatty acids and inhibits macrophage inflammatory mediators via the ERK pathway. PLoS One 8:e74875.

Laukens D, Georges M, Libioulle C, Sandor C, Mni M, Vander Cruyssen B, Peeters H, Elewaut D, and De Vos M (2010) Evidence for significant overlap between common risk variants for Crohn's disease and ankylosing spondylitis. PLoS One 5:e13795.

Lin Y-N, Roy A, Yan W, Burns KH, and Matzuk MM (2007) Loss of zona pellucida binding proteins in the acrosomal matrix disrupts acrosome biogenesis and sperm morphogenesis. Mol Cell Biol 27:6794-6805.

Löser S, Gregory LG, Zhang Y, Schaefer K, Walker SA, Buckley J, Denney L, Dean $\mathrm{CH}$, Cookson WOC, Moffatt MF, et al. (2017) Pulmonary ORMDL3 is critical for induction of Alternaria-induced allergic airways disease. J Allergy Clin Immunol 139:1496-1507.e3

Mells GF, Floyd JAB, Morley KI, Cordell HJ, Franklin CS, Shin S-Y, Heneghan MA, Neuberger JM, Donaldson PT, Day DB, et al.; UK PBC Consortium; Wellcome Trust Case Control Consortium 3 (2011) Genome-wide association study identifies 12 new susceptibility loci for primary biliary cirrhosis [published correction appears in Nat Genet (2011) 43:1164]. Nat Genet 43:329-332.

Miller M, Rosenthal P, Beppu A, Mueller JL, Hoffman HM, Tam AB, Doherty TA McGeough MD, Pena CA, Suzukawa M, et al. (2014) ORMDL3 transgenic mice have increased airway remodeling and airway responsiveness characteristic of asthma. J Immunol 192:3475-3487.

Miller M, Vuong C, Garcia MF, Rosenthal P, Das S, Weng N, Pham A, Kim YJ, and Broide DH (2018) Does reduced zona pellucida binding protein 2 (ZPBP2) expression on chromosome 17q21 protect against asthma? J Allergy Clin Immunol 142:706-709.e4.

Moffatt MF, Kabesch M, Liang L, Dixon AL, Strachan D, Heath S, Depner M, von Berg A, Bufe A, Rietschel E, et al. (2007) Genetic variants regulating ORMDL3 expression contribute to the risk of childhood asthma. Nature 448:470-473.

Moussette S, Al Tuwaijri A, Kohan-Ghadr H-R, Elzein S, Farias R, Bérubé J, Ho B, Laprise C, Goodyer CG, Rousseau S, et al. (2017) Role of DNA methylation in expression control of the IKZF3-GSDMA region in human epithelial cells. PLoS One 12:e0172707.

Paulenda T and Draber P (2016) The role of ORMDL proteins, guardians of cellular sphingolipids, in asthma. Allergy 71:918-930.

Rael EL and Lockey RF (2011) Interleukin-13 signaling and its role in asthma. World Allergy Organ J 4:54-64.

Saleh NM, Raj SM, Smyth DJ, Wallace C, Howson JMM, Bell L, Walker NM, Stevens HE, and Todd JA (2011) Genetic association analyses of atopic illness and proinflammatory cytokine genes with type 1 diabetes. Diabetes Metab Res Rev 27: 838-843.

Steinke JW and Borish L (2001) Th2 cytokines and asthma. Interleukin-4: its role in the pathogenesis of asthma, and targeting it for asthma treatment with interleukin-4 receptor antagonists. Respir Res 2:66-70.

Tomita K, Sakashita M, Hirota T, Tanaka S, Masuyama K, Yamada T, Fujieda S, Miyatake A, Hizawa N, Kubo M, et al. (2013) Variants in the 17q21 asthma susceptibility locus are associated with allergic rhinitis in the Japanese population. Allergy 68:92-100

Torabi F, Bogle OA, Estanyol JM, Oliva R, and Miller D (2017) Zona pellucidabinding protein 2 (ZPBP2) and several proteins containing BX7B motifs in human sperm may have hyaluronic acid binding or recognition properties. Mol Hum Reprod 23:803-816.

Torgerson DG, Ampleford EJ, Chiu GY, Gauderman WJ, Gignoux CR, Graves PE, Himes BE, Levin AM, Mathias RA, Hancock DB, et al.; Mexico City Childhood Asthma Study (MCAAS), Children's Health Study (CHS) and HARBORS study, Genetics of Asthma in Latino Americans (GALA) Study, Study of GenesEnvironment and Admixture in Latino Americans (GALA2) and Study of African Americans, Asthma, Genes \& Environments (SAGE), Childhood Asthma Research and Education (CARE) Network, Childhood Asthma Management Program 
(CAMP), Study of Asthma Phenotypes and Pharmacogenomic Interactions by Race-Ethnicity (SAPPHIRE), Genetic Research on Asthma in African Diaspora (GRAAD) Study (2011) Meta-analysis of genome-wide association studies of asthma in ethnically diverse North American populations. Nat Genet 43 $887-892$

Verlaan DJ, Berlivet S, Hunninghake GM, Madore A-M, Larivière M, Moussette S, Grundberg E, Kwan T, Ouimet M, Ge B, et al. (2009) Allele-specific chromatin remodeling in the ZPBP2/GSDMB/ORMDL3 locus associated with the risk of asthma and autoimmune disease. Am J Hum Genet 85:377-393.

Wan YI, Shrine NRG, Soler Artigas M, Wain LV, Blakey JD, Moffatt MF, Bush A Chung KF, Cookson WOCM, Strachan DP, et al.; Australian Asthma Genetic Consortium (2012) Genome-wide association study to identify genetic determinants of severe asthma. Thorax 67:762-768.

Williams TJ (2015) Eotaxin-1 (CCL11). Front Immunol 6:84

Worgall TS, Veerappan A, Sung B, Kim BI, Weiner E, Bholah R, Silver RB, Jiang X-C, and Worgall S (2013) Impaired sphingolipid synthesis in the respiratory tract induces airway hyperreactivity. Sci Transl Med 5:186ra67.

Youssef M, De Sanctis JB, Kanagaratham C, Tao S, Ahmed E, and Radzioch D (2020) Efficacy of Optimized Treatment Protocol Using LAU-7b Formulation Against
Ovalbumin (OVA) and House Dust Mite (HDM) -Induced Allergic Asthma in Atopic Hyperresponsive A/J Mice. Pharm Res 2:31-37.

Youssef M, De Sanctis JB, Shah J, Dumut DC, Hajduch M, Petrof BJ, and Radzioch D (2020) Age-Dependent Progression in Lung Pathophysiology Can Be Prevented by Restoring Fatty Acid and Ceramide Imbalance in Cystic Fibrosis. Lung, doi: 10.1007/s00408-020-00353-2.

Youssef M, Kanagaratham C, Saad MI, and Radzioch D (2016) Genetics of allergic asthma and current perspectives on therapeutic management, in Asthma - From Childhood Asthma to ACOS PhenotypesPereira C ed) pp 137-184, BoD - Books on Demand, Norderstedt, Germany.

Zhang Y, Willis-Owen SAG, Spiegel S, Lloyd CM, Moffatt MF, and Cookson WOCM (2019) The ORMDL3 asthma gene regulates ICAM1 and has multiple effects on cellular inflammation. Am J Respir Crit Care Med 199:478-488.

Address correspondence to: Dr. Danuta Radzioch, McGill University Health Center (RIMUHC), 1001 Decarie Blvd., Room EM3-3211, Montreal, QC H4A 3J1, Canada. E-mail: danuta.radzioch@mcgill.ca 